Molecular Genetics and Metabolism (2004) 81: S52-S57

\title{
Ammonia toxicity to the brain and creatine
}

\section{Claude Bachmann, Olivier Braissant, Anne-Marie Villard, Olivier Boulat and Hugues Henry.}

Laboratoire Central de Chimie Clinique, Centre Hospitalier Universitaire Vaudois, University of Lausanne, Switzerland

Running Title : Ammonia toxicity to the brain and creatine

Address for correspondence :

Prof. Claude Bachmann

LCC, CHUV

Bugnon 46

1011 Lausanne, Switzerland

Tel $\quad+41213144144$

FAX $\quad+41213144133$

email Claude. Bachmann@chuv.hospvd.ch

Key Words : Ammonia, Urea cycle, Nitrous oxide, Arginine, Creatine, Brain development, Axons, MAP-kinase, Glutathione 


\begin{abstract}
Symptoms of hyperammonemia are age-dependant; some are reversible. Multiple mechanisms are involved. Hyperammonemia increases the uptake of tryptophan into the brain by activation of the L-system carrier; brain glutamine plays a still undefined role. The uptake of tryptophan by the brain is enhanced if the levels of branched-chain amino acids competing with the other large neutral amino acids are low in plasma. Hyperammonemia increases the utilization of branched-chain amino acids in muscle when ketoglutarate is low; this is further enhanced with glutamine depletion (using phenylbutyrate). Anorexia, most likely a serotoninergic symptom, might further aggravate the deficiency of indispensable amino acids (branched-chain and arginine). The role of increased glutamine production in astrocytes and the excitotoxic and metabotropic effects of increased extracellular glutamate have been extensively described. They differ between models of acute and chronic hyperammonemia. Using an in vitro model of cultured embryonic rat brain cell aggregates, we studied the role of creatine. Cultures exposed to ammonia before maturation showed impaired cholinergic axonal growth accompanied by a decrease of creatine and phosphocreatine, a finding not seen in mature cultures. By using different antibodies we have shown that the phosphorylated form of the intermediate neurofilament protein is affected. Adding creatine to the culture medium partially prevents impairment of axonal growth. The presence of glia in the culture is a precondition for this protective effect. Adequate arginine substitution is essential in the treatment of urea cycle defects as creatine is inefficiently transported into the brain.
\end{abstract}




\section{Introduction}

Hyperammonemic disorders in pediatrics and especially urea cycle defects (UCD) present with both reversible and irreversible disabling symptoms, the mechanisms of which are not fully understood. In patients and in experimental animal models the various mechanisms are often additive or interacting, leading to a vicious cycle that renders a therapeutic intervention difficult and challenging or makes the interpretation of experimental results tedious. Therapeutic schemes and recommendations are useful as a starting point, but a better understanding is needed for the interpretation of the dietary history, symptoms and biochemical control data and, consequently, for timely changes in the treatment of an individual patient to prevent further irreversible damage. Our aim is to understand the main mechanisms of the irreversible, disabling effects of hyperammonemia on the patients during their development and especially those affecting the central nervous system (CNS) that lead to the poor long term prognosis of surviving patients even treated by using the actual conservative therapy [1].

\section{Ammonia, large neutral amino acids and glutamine}

The pathophysiologic mechanisms of toxicity of ammonia and its metabolites on the brain have been reviewed recently [2] [3] [4]. The effects of an acute increase in plasma ammonia should be distinguished, both clinically and experimentally, from chronic moderate increases [5]. Changes of metabolites other than ammonia in blood also affect the brain. As summarized by Gropman and Batshaw [6] the transport of tryptophan, the precursor of serotonin, from blood to brain, is increased in the hyperammonemic brain. This is caused by:

a) a decrease in large neutral amino acids, mainly branched-chain amino acids (BCAA), that compete with tryptophan for the usually saturated transporter (mainly system L1) of large neutral amino acids (LNAA). Hyperammonemia decreases BCAA in plasma and muscle as 
important nitrogen donors for glutamine synthetase. BCAA deficiency and to a lesser extent decreased levels of other proteogenic amino acids will hinder protein synthesis by increasing excessively insulin like growth factor (IGF) binding protein expression [7] [8] as compared to IGF and thus increase catabolism and ammonia load. The clinical observations by Maestri et al [9] indicate that the glutamine depletion effected by phenylbutyrate application adds to the decrease of BCAA. This is supported by experimental data [10]. It is not clear whether the beta oxidation of phenylbutyrate (medium chain acyl dehydrogenase) is enhancing the leucine oxidation in tissue.

b) an increased activity/density of the luminal transporter(s) of the brain microvessels.

The increase of tryptophan leads to an increased synthesis and release of serotonine and thus might induce anorexia[11,12]; this symptom often found in UCD patients renders them prone to chronic catabolism and malnutrition and thus to increased ammonia load resulting in a vicious cycle.

\section{Is glutamine in plasma and/or in the brain increasing tryptophan uptake at the blood brain barrier?}

There is confusion in case reports as to the comparative roles of plasma and brain glutamine. Is plasma glutamine solely a marker of the ammonia metabolism in the periphery or is it directly involved in the pathogenesis of neurologic symptoms?

Plasma glutamine can be viewed as the extracellular transport form of glutamate. The increase of plasma glutamine in UCD indicates that its production in tissue might be increased and that the capacity of the urea cycle in the liver, located near the portal vessels as first line of defense, is insufficient, but that the hepatic perivenous glutamine synthesis as second line of ammonia detoxication in the liver is functioning. This implies that splanchnic blood does not bypass the liver and that liver glutamate dehydrogenase is functioning normally. 
Glutamine in brain is formed mainly within astrocytes from ammonia and intermediates of glucose and fatty acids metabolism (octanoate 20\%; [13]). Unidirectional influx from the blood in anesthetized rats is only $5 \mathrm{nmol} / \mathrm{g}$ per minute as compared to a rate of glutamine synthesis within the brain in normal rats of $40 \mathrm{nmol} / \mathrm{g}$ per minute that can increase up to 200 $\mathrm{nmol} / \mathrm{g}[14]$.

Experimentally, an increased concentration of glutamine within brain microvessel cells enhances the transport of LNAA into the tissue. This is true (in bovine microcapillaries) for L- and for D-glutamine [11] [15] [2]. The use of inhibitors of glutathione synthesis (acivicin, methionine sulfoximine, or specifically buthionine sulfoximine) indicates that gammaglutamyl transfer, located at the luminal side of brain capillaries, plays a role in the transport of tryptophan [16] [2]. Other glutamine transport systems (system N, A and $\mathrm{B}^{0,+}[16]$ ) are active at the abluminal side of the blood brain barrier.

As D-glutamine is a poor substrate for system- $\mathrm{L}$ and as - to our knowledge - no racemase converting D- into L-glutamine has been shown in mammals, the increased transport of LNAA under hyperammonemic conditions does not simply occur by exchange with glutamine catalyzed by the system- $\mathrm{L}$ transporter(s) but by a mechanism that is not fully understood.

Since there is a net arterio-venous difference in chronic experimental hyperammonemia indicating that transport of glutamine out of the brain prevails, it is likely that the concentration of ammonia in the brain is more relevant than that in plasma for the stimulation of tryptophan uptake into the brain. This hypothesis is supported by the lack of correlation between the plasma concentrations of glutamine and tryptophan in urease-injected rats [17].

Interestingly, Hammond et al. [18] reported increased concentrations of gammaglutamylglutamine in plasma and CSF taken from hyperammonemic patients. It cannot be excluded that in CSF this compound originates from the gamma-glutamyl cycle expressed in 
the choroid plexus. It points to the fact that at high concentrations L-glutamine might be an acceptor substrate for gamma-glutamyl transferase, provided glutathione is not limiting.

In models of brain edema, where lethal doses of ammonia are administered, glial fibrillary acidic protein (GFAP) is reduced [19] and glutamine is increased in brain [20]; this latter is preceded by an increase in cerebral blow flow [21]. Whether nitrous oxide (NO) production in brain capillary endothelia plays a role is not clear. NO could also originate from brain cells, as the arginine recycling enzymes are induced in astrocytes by ammonium [22]. The constitutive NO synthetase of brain (nNOS) as well as the NO cycle enzyme argininosuccinate synthetase are not expressed in astrocytes of normal adult rats; the other players in the NO cycle argininosuccinate lyase, arginase2 and the cationic amino acid transporter CAT1 are found in neurons and glial cells, but expressed to a variable degree and dependent on location. CAT2B and CAT3 are not expressed in astrocytes [22] [23]. If NO is formed in astrocytes at the blood brain barrier then the reaction is catalyzed by the inducible NOS (if present). In contrast to most animal models plasma arginine is very low in patients with urea cycle defects (e.g. AL deficiency). One might thus consider that at low brain arginine concentrations the stimulated nNOS in neurons might mainly produce $\mathrm{O}_{2}^{-}$that combines with NO to form the highly toxic peroxynitrite.

\section{Glutamate}

Glutamate is not increased in astrocytes in hyperammonemia because glutamine formation from ammonium and glutamate prevails over glutamate synthesis. Chronic moderate hyperammonemia impairs the activation of NMDA receptors and leads to a reduced reuptake of extracellular glutamate [25]. After prior exposure to ammonia, rat astrocytes in primary culture show a reduced production of free radicals after ammonium load as compared to that of an initial acute challenge [26]; cGMP formation in cerebellar neurons appears to be 
stimulated and cGMP concentrations in brain microdialysate of chronic moderately hyperammonemic rats were found lower than in controls [25]. Neither neuronal NOS nor NMDA receptor activation was affected under these conditions.

In contrast, acute hyperammonemia leads to excessive activation of NMDA receptors, increased intracellular $\mathrm{Ca}++$, which bound to calmodulin, stimulates NOS; NO activates the guanylate cyclase (cerebellum) [27,28]. This difference between chronic and acute hyperammonemia might, at least in part, be explained by the neuromodulation of glutamate receptors by glutathione (for review see [29]). In astrocyte cultures glutathione is increased by ammonium in a dose and time dependant way [30]. It is readily released from astrocytes [31].

\section{Creatine}

Animal experiments are plagued by the multiple variables that cannot be controlled (effects of the decrease of intake of chow and water in hyperammonemic rats, serotoninergic and more direct effects of ammonia mentioned above). To investigate the irreversible effects of ammonia increase we used aggregates of rat embryonic brain cells cultured in serum-free chemically defined medium [32] [33]. This method allows the culture of astrocytes, neurons, oligodendrocytes and microglia together and thus also to study intercellular interactions. Using this model, we showed that ammonia impairs axonal growth as well as medium weight neurofilament (NF-M ) expression and phosphorylation [34]. We have further demonstrated that ammonia impairs axons only during their maturation, but not when neurons have gone through synaptogenesis [34].

We have shown that exposing brain cells to ammonia may alter the recycling of arginine, which plays key roles in CNS not only as substrate for protein synthesis or precursor of NO, but also as substrate for creatine synthesis; there is substantial evidence that creatine is 
essential for axonal elongation [24] [34]. We have thus tested whether creatine metabolism is altered by ammonia, and shown that indeed $\mathrm{NH}_{4} \mathrm{Cl}$ exposure of brain cell aggregates leads to a decrease of intracellular creatine and phosphocreatine [34]. When the aggregate cultures are co-treated with creatine $(1 \mathrm{mM})$ during $\mathrm{NH}_{4} \mathrm{Cl}(5 \mathrm{mM})$ exposure, creatine protects the axons from growth impairment to a certain extent, in a glial cell dependent manner [34].

For a better understanding of the protective effect of creatine, we have analyzed the gene expression of the creatine synthesis pathway and transporter in the different cell types of CNS. This was achieved by in situ hybridization [35] and immunohistochemistry with specific antibodies for L-arginine:glycine amidinotransferase (AGAT, supplied by O.Speer and T.Wallimann), as well as for S-adenosyl-L-methionine:N-guanidinoacetate methyltransferase (GAMT) and for the creatine transporter (CRT1), developed in our laboratory. The anti-CRT1 antibody was directed against amino acids 15-29 of the sequence [36]. AGAT and GAMT are expressed in astrocytes, oligodendrocytes and neurons whereas CRT1 is present in neurons and oligodendrocytes but cannot be detected in astrocytes, including those contacting capillaries [35]. Thus CRT1 in the brain may function predominantly as an intercellular transporter and not for bulk transport from the blood: the brain may thus depend to a large extent on local de novo synthesis of creatine. This is in accordance with the clinical observation in congenital defects of creatine synthesis or transport or in ornithine aminotransferase deficiency where supplementation of creatine at high doses over a prolonged period is necessary to increase its brain concentration to some extent without reaching full restoration.

When aggregates were exposed to ammonia a moderate induction of CRT1 was observed in astrocytes, including those resembling Alzheimer type II cells. The normally high expression of CRT1 in oligodendrocytes was decreased by ammonia exposure. 
As we have shown that exposing brain cell aggregates to ammonia induces arginine recycling enzymes in astrocytes [24], and decrease intracellular levels of creatine and phosphocreatine [34] , we further investigated the changes in intracellular concentrations of arginine as one key substrate of creatine synthesis as well as of other amino acids in brain cell aggregates exposed to ammonia and/or creatine (13 days of culture, exposure from days 5 to 13; Table 1). The concentration of arginine (substrate for AGAT) is increased by $1 \mathrm{mM}$ ammonia exposure and remains on the same plateau level at higher concentrations (2.5 and $5 \mathrm{mM}$ ), while ornithine (product of AGAT) increases after exposure to $5 \mathrm{mM}$ ammonia. In contrast the concentration of most essential amino acids drops at that level of ammonia as compared to lower ammonia concentrations except for branched chain amino acids. Levels of glutamate and its precursors, proline and alanine, decrease. The concentrations of most of the amino acids are lower with creatine co-treatment (Table 1). Interestingly, for arginine the ammonia and creatine effects are non-additive. It is not clear whether at high ammonia concentrations there is competition between arginine and creatine for their transporters.

Brain cell cytoskeletal elements have recently been found to be altered under hyperammonemic conditions. The phosphorylation of microtubule associated protein 2 (MAP-2) is altered in the brain of hyperammonemic rats without liver failure with consequences on binding capacity to tubulin and its polymerisation [37] [38] [39] [40]. Furthermore we showed that the phosphorylation of NF-M is decreased in aggregates exposed to ammonia [34]. These findings must be viewed in the context of a more general alteration of protein phosphorylation as summarized recently by Corbalan et al. [41].

As mitogen activated protein kinases (MAPK) are the main cascades linking extracellular signals to neuronal differentiation and axonal elongation, we started to investigate the 
regulation of MAPK pathways in brain cell aggregates exposed to ammonia. Preliminary western blot data have been obtained, showing that upon exposure to ammonia the phosphorylation of Erk1/Erk2 increases in homogenates of mixed cell cultures, but decreases in neuron-enriched aggregates. The level of phosphorylation of SAPK/JNK is decreased both in mixed cell and neuron enriched cultures [42]. Further work is needed at the histological level to assess whether these changes vary uniformly among the cell types involved, or if these ammonia effects vary between glial cells and neurons.

As to the link to ammonia and creatine it is of interest that the neuronal calcium-dependant reactive oxygen species production depends on a functioning MAPK pathway; this effect can be blocked by nerve growth factor application [43]. However whether this applies to NO and/or peroxynitrite (mentioned above) is not known. The Erk1/Erk2 and SAPK/JNK signal transduction cascades also play a role in gene transcription following NMDA receptor stimulation [44] [45]. In vivo, 5- $\mathrm{HT}_{1 \mathrm{~A}}$ post-synaptic receptor activation reduces phospho-Erk2 levels in the hippocampus, but not in the striatum or frontal brain. Interestingly, there is no effect on SAPK/JNK or p38 MAPK [46].

\section{Conclusions}

In clinical practice the first step in the treatment of hyperammonemic crises is to stop the nitrogen load, be it nutritional in origin and/or due to catabolism. When managing patients with diagnosed UCD a depletion of arginine and of indispensable amino acids should be prevented by replacing if needed a part of natural protein by a special mixture of essential amino acids with high branched chain and low aromatic acids. This will not only improve the quality of the limited natural protein ingested but also correct any imbalance with its consequences to the brain. 
Despite the protective action of creatine on axonal development, supplementing creatine as such does not seem to be promising, as the brain seems well protected against excessive creatine influx. However its precursor, L-arginine, which is low in the plasma of untreated patients with UCD (except for arginase deficiency), must be supplemented in sufficient amounts. It is readily transported into the brain and needed for creatine, protein and NO synthesis.

Acknowledgement: Supported by Swiss National Science Foundation (grants n 3100-63892 and 3100A0-100778). 


\section{References}

1 C. Bachmann, Outcome and survival of 88 patients with urea cycle disorders: a retrospective evaluation., Eur. J. Pediatr. 162 (2003) 410-416.

2 C. Bachmann, Mechanisms of hyperammonemia, Clin. Chem. Lab. Med. 40 (2002) 653662.

3 V. Felipo, R.F. Butterworth, Neurobiology of ammonia, Progr. Neurobiol. 67 (2002) 259279.

4 R.F. Butterworth, Pathophysiology of hepatic encephalopathy: a new look at ammonia, Metab. Brain Dis. 17 (2002) 221-227.

5 R.F. Butterworth, Glutamate transporter and receptor function in disorders of ammonia metabolism, MRDD Res. Rev. 7 (2001) 276-279.

6 A.L. Gropman, M.L. Batshaw, Cognitive Outcome in Urea Cycle Disorders, Mol. Genet. Metab. (2003) in press.

7 A. Bruhat, C. Jousse, P. Fafournoux, Amino acid limitation regulates gene expression., Proc. Nutr. Soc. 58 (1999) 625-632.

8 Y. Endo, Z. Fu, K. Abe, S. Arai, H. Kato, Dietary protein quantity and quality affect rat hepatic gene expression., J. Nutr. 132 (2002) 3632-3637.

9 N.E. Maestri, D.B. Clissold, S.W. Brusilow, Long-term survival of patients with argininosuccinate synthetase deficiency, J. Pediatr. 127 (1995) 929-935.

10 D. Darmaun, S. Welch, A. Rini, B.K. Sager, A. Altomare, M.W. Haymond, Phenylbutyrate-induced glutamine depletion in humans: effect on leucine metabolism, Am. J. Physiol. 274 (1998) E801-807.

11 C. Bachmann, J.P. Colombo, Increased tryptophan uptake into the brain in hyperammonemia., Life Sci. 33 (1983) 2417-2424.

12 C. Bachmann, J.P. Colombo, Increase of tryptophan and 5-hydroxyindole acetic acid in the brain of ornithine carbamoyltransferase deficient sparse-fur mice, Pediatr. Res. 18 (1984) 372-375.

13 D. Ebert, R.G. Haller, M.E. Walton, Energy contribution of octanoate to intact rat brain metabolism measured by 13C nuclear magnetic resonance spectroscopy, J. Neurosci. 23 (2003) 5928-5935. 
14 J. Xiang, S.R. Ennis, G.E. Abdelkarim, M. Fujisawa, N. Kawai, R.F. Keep, Glutamine transport at the blood-brain and blood-cerebrospinal fluid barriers, Neurochem. Int. 43 (2003) 279-288.

15 M. Gorgievski-Hrisoho, J.P. Colombo, C. Bachmann, Stimulation of tryptophan uptake into brain microvessels by D-glutamine, Brain Res. 367 (1986) 395-397.

16 W.J. Lee, R.A. Hawkins, J.R. Vina, D.R. Peterson, Glutamine transport by the bloodbrain barrier: a possible mechanism for nitrogen removal., Am. J. Physiol. 274 (1998) C1101-1107.

17 M.L. Batshaw, S.L. Hyman, E.D. Mellits, G.H. Thomas, R. DeMuro, J.T. Coyle, Behavioral and neurotransmitter changes in the urease-infused rat: a model of congenital hyperammonemia, Pediat. Res. 20 (1986) 1310-1315.

18 J.W. Hammond, M. Potter, R. Truscott, B. Wilcken, Gamma-Glutamylglutamine identified in plasma and cerebrospinal fluid from hyperammonaemic patients, Clin. Chim. Acta 194 (1990) 173-183.

19 M. Belanger, P. Desjardins, N. Chatauret, R.F. Butterworth, Loss of expression of glial fibrillary acidic protein in acute hyperammonemia, Neurochem. Int. 41 (2002) 155-160.

20 A.J. Cooper, Role of glutamine in cerebral nitrogen metabolism and ammonia neurotoxicity, MRDD Res. Rev. 7 (2001) 280-286.

21 F.S. Larsen, J. Gottstein, A.T. Blei, Cerebral hyperemia and nitric oxide synthase in rats with ammonia-induced brain edema, J. Hepatol. 34 (2001) 548-554.

22 O. Braissant, T. Gotoh, M. Loup, M. Mori, C. Bachmann, L-arginine uptake, the citrulline-NO cycle and arginase II in the rat brain: an in situ hybridization study, Brain Res. Mol. Brain Res. 70 (1999) 231-241.

23 O. Braissant, T. Gotoh, M. Loup, M. Mori, C. Bachmann, Differential expression of the cationic amino acid transporter 2(B) in the adult rat brain, Brain Res. Mol. Brain Res. 91 (2001) 189-195.

24 O. Braissant, P. Honegger, M. Loup, K. Iwase, M. Takiguchi, C. Bachmann, Hyperammonemia: regulation of argininosuccinate synthetase and argininosuccinate lyase genes in aggregating cell cultures of fetal rat brain, Neurosci. Lett. 266 (1999) 8992.

25 C. Hermenegildo, C. Montoliu, M. Llansola, M.D. Munoz, J.M. Gaztelu, M.D. Minana, V. Felipo, Chronic hyperammonemia impairs the glutamate-nitric oxide-cyclic GMP 
pathway in cerebellar neurons in culture and in the rat in vivo, Eur. J. Neurosci. 10 (1998) 3201-3209.

26 P. Monfort, C. Montoliu, C. Hermenegildo, M. Munoz, V. Felipo, Differential effects of acute and chronic hyperammonemia on signal transduction pathways associated to NMDA receptors, Neurochem. Int. 37 (2000) 249-253.

27 E. Kosenko, Y. Kaminsky, E. Grau, M.D. Minana, G. Marcaida, S. Grisolia, V. Felipo, Brain ATP depletion induced by acute ammonia intoxication in rats is mediated by activation of the NMDA receptor and $\mathrm{Na}^{+}, \mathrm{K}(+)$-ATPase, J. Neurochem. 63 (1994) 21722178.

28 E. Kosenko, Y. Kaminsky, S. I.G., V. Felipo, Alteration of mitochondrial calcium homeostasis by ammonia-induced activation of NMDA receptors in rat brain in vivo., Brain Res. 880 (2000) 139-146.

29 S.S. Oja, R. Janaky, V. Varga, P. Saransaari, Modulation of glutamate receptor functions by glutathione, Neurochem. Int. 37 (2000) 299-306.

30 C.R. Murthy, A.S. Bender, R.S. Dombro, G. Bai, M.D. Norenberg, Elevation of glutathione levels by ammonium ions in primary cultures of rat astrocytes, Neurochem. Int. 37 (2000) 255-268.

31 M. Yudkoff, D. Pleasure, L. Cregar, Z.P. Lin, I. Nissim, J. Stern, Glutathione turnover in cultured astrocytes: studies with [15N]glutamate, J. Neurochem. 55 (1990) 137-145.

32 P. Honegger, F. Monnet-Tschudi, Aggregating neural cell culture, in: S. Fedoroff,A. Richardson (Eds.), Protocols for neural cell culture, Humana Press, Totowa,NJ, 1997, pp. 25-49.

33 C. Bachmann, H. Henry, O. Braissant, From Arginine to Creatine: Regional and Cellular Gene Expression of Enzymes and Transporters Linking Nitrogen and Energy Metabolism in Brain., in: C. Bachmann,B. Koletzko (Eds.), Genetic Expression and Nutrition, Nestlé Nutrition Workshop Series. 50, Nestec Ltd./Lipincott Williams \& Wilkins, Vevey/Philadelphia, 2003, pp. 153-165.

34 O. Braissant, H. Henry, A.M. Villard, M.G. Zurich, M. Loup, B. Eilers, G. Parlascino, E. Matter, O. Boulat, P. Honegger, C. Bachmann, Ammonium-induced impairment of axonal growth is prevented through glial creatine, J. Neurosci. 22 (2002) 9810-9820.

35 O. Braissant, H. Henry, M. Loup, B. Eilers, C. Bachmann, Endogenous synthesis and transport of creatine in the rat brain: an in situ hybridization study, Brain Res. Mol. Brain Res. 86 (2001) 193-201. 
36 H. Henry, O. Braissant, B. Eilers, E. Matter, C. Bachmann, Identification and expression of the rat brain creatine transporter 1 produced by alternate splicing, submitted (2003)

37 M.D. Minana, V. Felipo, R. Wallace, S. Grisolia, High ammonia levels in brain induce tubulin in cerebrum but not in cerebellum., J. Neurochem. 51 (1988) 1839-1842.

38 V. Felipo, E. Grau, M.D. Minana, S. Grisolia, Activation of NMDA receptor mediates the toxicity of ammonia and the effects of ammonia on the microtubule-associated protein MAP-2., Adv. Exp. Med. Biol. 341 (1993) 83-93.

39 V. Felipo, E. Grau, M.D. Minana, S. Grisolia, Hyperammonemia decreases proteinkinase-C-dependent phosphorylation of microtubule-associated protein 2 and increases its binding to tubulin., Eur. J. Biochem. 214 (1993) 243-249.

40 R. Saez, M. Llansola, V. Felipo, Chronic exposure to ammonia alters pathways modulating phosphorylation of microtubule-associated protein 2 in cerebellar neurons in culture., J. Neurochem. 73 (1999) 2555-2562.

41 R. Corbalan, M. Hernandez_Viadel, M. Llansola, C. Montoliu, V. Felipo, Chronic hyperammonemia alters protein phosphorylation and glutamate receptor-associated signal transduction in brain, Neurochem. Int. 41 (2002) 103-108.

42 A.M. Villard, P. Honegger, C. Bachmann, O. Braissant, Axonal growth impairment by ammonium and protection through glial creatine. Involvement of MAPK pathways, USGEB, Davos, Switzerland, 19-21 March 2003.

43 L.L. Dugan, D.J. Creedon, E.M. Johnson, D.M. Holtzman, Rapid suppression of free radical formation by nerve growth factor involves the mitogen-activated protein kinase pathway, Proc. Natl. Acad. Sci. U S A 94 (1997) 4086-4091.

44 Z. Xia, H. Dudek, C.K. Miranti, M.E. Greenberg, Calcium influx via the NMDA receptor induces immediate early gene transcription by a MAP kinase/ERK-dependent mechanism, J. Neurosci. 16 (1996) 5425-5436.

45 T. Borsello, K. Croquelois, J.P. Hornung, P.G. Clarke, N-methyl-d-aspartate-triggered neuronal death in organotypic hippocampal cultures is endocytic, autophagic and mediated by the c-Jun N-terminal kinase pathway, Eur. J. Neurosci. 18 (2003) 473-485.

46 J. Chen, C. Shen, E. Meller, 5-HT1A receptor-mediated regulation of mitogen-activated protein kinase phosphorylation in rat brain, Eur. J. Pharmacol. 452 (2002) 155-162. 
Table 1. Intracellular amino acid concentrations ( $\mu \mathrm{mol} / \mathrm{g}$ protein) of mixed cultured aggregates harvested at 13 days after being exposed from days 5 to 13 to ammonia and / or creatine as indicated on top.

\section{Legend to Table 1}

Factorial analysis ( $\mathrm{n}=3$ per condition) shows a highly significant ammonia effect $(\mathrm{p}<0.001)$ for all amino acids except for arginine, glutamine, serine, aspartate with significance of $0.001<\mathrm{p}<0.01$ and not significant for histidine, valine, ornithine, GABA, cysteine and citrulline. The creatine effect was highly significant $(\mathrm{p}<0.001)$ for all amino acids except arginine, GABA, glycine and serine reaching a level of $0.001<\mathrm{p}<0.01$; not significant: ornithine, cysteine and citrulline. Significant interactions, i.e. non-additive effects of ammonia and creatine, were found for leucine and asparagine $(\mathrm{p}<0.05)$, cystathionine $(\mathrm{p}=0.026)$ and especially arginine $(\mathrm{p}=0.020)$. 


\begin{tabular}{|c|c|c|c|c|c|c|c|c|}
\hline Creatine (mM) & 0 & 0 & 0 & 0 & 1 & 1 & 1 & 1 \\
\hline $\mathrm{NH}_{4}^{+}(\mathrm{mM})$ & 0 & 1 & 2.5 & 5 & 0 & 1 & 2.5 & 5 \\
\hline Phenylalanine & $8.8 \pm 0.86$ & $9.0 \pm 1.47$ & $9.0 \pm 0.18$ & $7.1 \pm 0.45$ & $7.6 \pm 0.24$ & $7.2 \pm 0.25$ & $6.5 \pm 0.56$ & $5.6 \pm 0.15$ \\
\hline Tyrosine & $9.8 \pm 0.93$ & $10.2 \pm 1.48$ & $9.9 \pm 0.36$ & $7.8 \pm 0.07$ & $8.6 \pm 0.3$ & $8.0 \pm 0.2$ & $7.5 \pm 0.45$ & $6.1 \pm 0.12$ \\
\hline Methionine & $2.7 \pm 0.26$ & $2.5 \pm 0.46$ & $2.5 \pm 0.27$ & $2.1 \pm 0.19$ & $2.4 \pm 0.01$ & $2.2 \pm 0.09$ & $2.0 \pm 0.09$ & $1.7 \pm 0.16$ \\
\hline Leucine & $5.9 \pm 0.54$ & $6.9 \pm 1.45$ & $8.0 \pm 0.18$ & $9.4 \pm 0.19$ & $5.4 \pm 0.34$ & $5.6 \pm 0.2$ & $6.1 \pm 0.4$ & $6.8 \pm 0.23$ \\
\hline Isoleucine & $6.1 \pm 0.61$ & $7.4 \pm 1.6$ & $8.2 \pm 0.23$ & $9.1 \pm 0.24$ & $5.7 \pm 0.32$ & $5.9 \pm 0.21$ & $6.3 \pm 0.37$ & $6.8 \pm 0.29$ \\
\hline Histidine & $4.2 \pm 0.57$ & $4.6 \pm 0.78$ & $4.5 \pm 0.54$ & $4.1 \pm 0.29$ & $3.9 \pm 0.39$ & $3.6 \pm 0.17$ & $3.4 \pm 0.33$ & $2.9 \pm 0.13$ \\
\hline Valine & $9.3 \pm 0.75$ & $10.3 \pm 1.7$ & $10.9 \pm 0.35$ & $10.8 \pm 0.31$ & $8.4 \pm 0.31$ & $8.2 \pm 0.03$ & $8.3 \pm 0.59$ & $8.0 \pm 0.2$ \\
\hline Threonine & $46.5 \pm 6.15$ & $46.6 \pm 5.73$ & $42.5 \pm 2.15$ & $32.4 \pm 0.82$ & $37.4 \pm 1.75$ & $35.2 \pm 1.09$ & $29.4 \pm 2.39$ & $23.9 \pm 0.84$ \\
\hline Arginine & $5.4 \pm 0.33$ & $6.9 \pm 0.78$ & $7.3 \pm 0.47$ & $7.3 \pm 0.27$ & $6.0 \pm 0.68$ & $6.4 \pm 0.16$ & $6.7 \pm 0.46$ & $6.0 \pm 0.32$ \\
\hline Lysine & $27.9 \pm 3.34$ & $30.7 \pm 2.85$ & $29.1 \pm 1.83$ & $21.5 \pm 0.48$ & $25.6 \pm 1.4$ & $24.7 \pm 0.54$ & $22.8 \pm 1.9$ & $17.5 \pm 0.24$ \\
\hline Ornithine & $1.0 \pm 0.14$ & $1.3 \pm 0.48$ & $1.1 \pm 0.05$ & $2.7 \pm 2.85$ & $1.2 \pm 0.54$ & $0.8 \pm 0.1$ & $1.2 \pm 0.97$ & $0.9 \pm 0.42$ \\
\hline Proline & $22.2 \pm 6.14$ & $19.8 \pm 1.36$ & $18.5 \pm 1.41$ & $10.5 \pm 0.78$ & $14.7 \pm 0.24$ & $13.0 \pm 0.9$ & $11.4 \pm 1.28$ & $8.8 \pm 0.6$ \\
\hline Glutamate & $94.9 \pm 12.7$ & $104 . \pm 11.5$ & $93.1 \pm 9.19$ & $38.1 \pm 0.37$ & $83.9 \pm 4.81$ & $85.7 \pm 0.76$ & $69.3 \pm 6.56$ & $32.9 \pm 1.03$ \\
\hline Glutamine & $286 \pm 34.2$ & $333 \pm 34$ & $354 \pm 15.3$ & $289 \pm 13.1$ & $246 \pm 9.2$ & $256 \pm 7.1$ & $258 \pm 18$ & $228 \pm 4.4$ \\
\hline$\gamma$-Aminobutyrate & $40.8 \pm 10.76$ & $42.1 \pm 5.05$ & $43.8 \pm 2.4$ & $36 \pm 3.93$ & $36.1 \pm 1.77$ & $34.4 \pm 2.93$ & $33.3 \pm 4.09$ & $28.2 \pm 3.07$ \\
\hline Glycine & $39.5 \pm 4.73$ & $38.2 \pm 4.37$ & $30.1 \pm 1.91$ & $22.8 \pm 4.29$ & $37.1 \pm 2.4$ & $33.3 \pm 1.45$ & $24.1 \pm 2.44$ & $16.3 \pm 0.83$ \\
\hline Taurine & $226 \pm 37.1$ & $256 \pm 23.2$ & $262 \pm 11.7$ & $184 \pm 8.9$ & $194 \pm 4.6$ & $193 \pm 4.3$ & $187 \pm 14.6$ & $145 \pm 12.2$ \\
\hline Cysteine & $0.5 \pm 0.07$ & $0.4 \pm 0.16$ & $0.4 \pm 0.23$ & $0.6 \pm 0.17$ & $0.4 \pm 0.2$ & $0.4 \pm 0.27$ & $0.4 \pm 0.09$ & $0.6 \pm 0.04$ \\
\hline Cystathionine & $11.5 \pm 1.56$ & $18.0 \pm 3.02$ & $19.0 \pm 1.77$ & $13.9 \pm 0.54$ & $8.2 \pm 0.95$ & $10.4 \pm 1.38$ & $9.7 \pm 0.79$ & $7.0 \pm 0.25$ \\
\hline Serine & $9.2 \pm 1.91$ & $11.4 \pm 2.44$ & $11.7 \pm 0.93$ & $20.9 \pm 8.17$ & $9.7 \pm 1.71$ & $10 \pm 0.85$ & $10.8 \pm 2.35$ & $11.0 \pm 1.69$ \\
\hline Alanine & $17.7 \pm 2$ & $17.1 \pm 2.12$ & $13.9 \pm 1.98$ & $6.7 \pm 3.14$ & $12.7 \pm 0.6$ & $11.5 \pm 0.72$ & $8.0 \pm 1.55$ & $4.2 \pm 0.2$ \\
\hline Aspartate & $12.1 \pm 2.32$ & $11.1 \pm 1.79$ & $11.6 \pm 0.36$ & $8.1 \pm 1.81$ & $8.9 \pm 0.68$ & $7.7 \pm 1.28$ & $7.8 \pm 1.88$ & $5.1 \pm 0.46$ \\
\hline Asparagine & $3.4 \pm 0.46$ & $3.2 \pm 0.18$ & $2.7 \pm 0.21$ & $1.5 \pm 0.28$ & $2.3 \pm 0.14$ & $2 \pm 0.03$ & $1.4 \pm 0.15$ & $1.0 \pm 0.26$ \\
\hline
\end{tabular}

